\title{
Effects of Motor Imagery Techniques in Children with Spastic Cerebral Palsy
}

\author{
Breera Amjad ${ }^{1 *}$, Muhammad Asif ${ }^{2}$, Erum Tanveer ${ }^{3}$, Abdul Rashad ${ }^{4}$, Heba Haider ${ }^{5}$, M Faizan Hassan ${ }^{6}$, Shujaat \\ Hussain Memon ${ }^{7}$, Hina Rathi ${ }^{8}$ and Kanza Masood ${ }^{9}$
}

${ }^{1}$ Senior Lecturer, Ripah International University, Faisalabad Campus, Pakistan

${ }^{2}$ Principal, Professor, Isra Institute of Rehabilitation Science, Isra University

${ }^{3}$ Principal \& Assistant Professor, United College of Physical Therapy

${ }^{4}$ Senior Lecturer, United College of Physical Therapy

${ }^{5}$ Physical Therapist, Mamji Hospital

${ }^{6}$ Physical Therapist, Ziauddin University

${ }^{7}$ Principal \& Assistant Professor, Hyderabad Institute of Medical \& Allied Sciences

${ }^{8}$ Assistant Professor, Suleman Roshan College of Physiotherapy and Rehabilitation Sciences

${ }^{9}$ Lecturer, University of Lahore, Sarghoda

Submission: July 15, 2019; Published: July 25, 2019

*Corresponding author: Breera Amjad, Senior Lecturer, Ripah International University, Faisalabad Campus, Pakistan

\begin{abstract}
Aim of Study: To explore the effects of motor imagery techniques on the balance and gross motor functions of Spastic Cerebral Palsy children.

Study Design: Randomized controlled trial RCT, was type of study.

Study Settings: Outpatient department of Physical Therapy at National Institute of Rehabilitation Medicine (NIRM) Islamabad.

Subjects: Twenty-two individuals with Spastic Cerebral Palsy were selected for the study.

Interventions of Study: Among study sample groups, interventional group did motor imagery techniques involving imagining different movements of body for 15 minutes surrounded with conventional physical therapy training for 30 minutes session (45 min./day, 3 sessions/ week), the control group executed conventional physical therapy session only (30 min./day, 3 times/week).
\end{abstract}

Outcome measures: Gross motor functional capacity and balance were measured by the Modified Functional Reach Test, Timed Up-and-Go Test and Gross Motor Functional Measure scale (GMFM-IS66).

Results: All measurements improved significantly compared with baseline values in the experimental group. In the control group, there were significant improvements in all parameters except the GMFM-IS66.

Conclusion: Balance training along with motor imagery techniques increases the balance and motor planning capabilities of spastic cerebral palsy children considerably enhanced than conventional physical therapy alone in cerebral palsy.

Abbreviations: NIRM: National Institute of Rehabilitation Medicine; GMFM: Gross Motor Functional Measure scale; MI: Motor Imagery; MP: Motor Performance; FMRI: Functional Magnetic Resonance Imaging; TUG: Time Up and Go; MFR: Modified Functional Reach

\section{Introduction}

Cerebral palsy is not an ailment, its impairment. Cerebral palsy is somewhat a clinical explanation and do not identify the nature of illness as the term indicates nothing regarding etiology, pathology or expected recovery time. The brain insults are important for cerebral palsy and can happen at any moment in the beginning of 1st trimester of infancy; so, it is a broad term that covers a lot of etiologies and pathologies and its clinical details are manifold. So, cerebral palsy is a clinical syndrome that presents as a disorder in the development of postures, balance, coordination and movements due to lesion of immature brain occurring in the foetal period or infancy. The appearance of cerebral palsy can be comprehensive, psychological and physical disability or secluded instabilities in gait, perception, development or perception. The result is motor or sensory 
nerve disorders and the incapability to accomplish many tasks in everyday life and participation restriction. CP causes damage to motor or sensory nerves, functional limitation and impaired performance of daily life activities [1]. Cerebral palsy patients do lack in motor planning and execution of motor task. Motor dysfunction in cerebral palsy is usually associated with disabilities in sensory consolidation and stability, weak muscular contractions, weak or totally absence of motor planning which is the prerequisite of a motor task execution.

Moreover, simultaneous stimulation of prime movers and its associated opponent muscles, absence of particular regulation of movement in organisms, and reduced anaerobic muscle capacity and activity cause impaired functions and tasks restriction. Patients with this neurological disorder usually have worse dynamic and static balance. Overhaul services have commonly been ignored. Generally, exist inside the well-being sector. It is especially because of deficiency of complete guidelines. Cerebral palsy rehabilitation centers are very necessary to combat this deficiency. Even many clinical institutes give proper guidelines and treatments for cerebral palsy but still it is deficient and oldfashioned. Even patients can expire due to the devastating effect of bodily impairment, but it is in very exceptional and intensive cases. New treatments need to be established in response to impairments. So, this study is going to evaluate a unique and rare technique to treat and rehab gross motor manifestations especially of motor planning deficit and balance impairments in cerebral palsy. During the previous decade, the usage of databased interventions in cerebral palsy regimen has moderately increased and researchers have struggled to evolve further productive interventions to raise the standards of living of these individuals and their households.

A latest analysis has investigated that interventions established on motor learning improves functional levels in children with cerebral palsy [2]. Therefore, using Motor Imagery Techniques in CP might be a promising technique to ensure improvement in motor planning deficit. Motor imagery is the cognitive presentation of movement in the absence of any bodily movement. Profuse verification on the beneficial influence of motor imagery implementation on motor presentation and training in sportsperson, fit people, and individuals having neurological problems (e.g., stroke, spinal cord injury, Parkinson's) has brought out. Imagery refers to the creation or re-creation of any practice in the mind like auditory, visual, olfactory, kinesthetic, organic. Specifically; motor imagery (MI) is the mental representation of movement without any body movement. It is a complex mental operation that is selfgenerated using sensory and perceptual processes, enabling the reactivation of specific motor actions within working memory. Therefore, sensory-perceptual, memory, and motor mechanisms are included in broader definitions of the term.

Motor imagery is the mental imagination of movement without any actual body movement. It is a mental activity by which an individual rehearses, or replicates a given action. It is mostly used in sport training as mental practice of action and also in neurological rehabilitation. Motor imagery is likely to develop more 'cognitive' aspects of motor behavior, and may, therefore, be helpful in promoting motor planning in patients with CP [3]. Several evidences show that motor imagery and actions are conveyed by the same brain circuits. For example, functional neuroimaging and transcranial Magnetic Stimulation studies demonstrate that the same brain structures are involved in action and imagining the same action. Functional brain imagines reviews have exhibited that many cortical as well as subcortical regions are triggered through definite motor performance, are equally triggered while imagination or cognitive execution of movements. Current verification shows that the primary motor cortex is associated with motor imagery procedure [4].

During motor imagery, many neural mechanisms are activated characterized by a marked elevation in tendinous reflexes of the limb part imagined moving, and by somatic variations which resembles with the level of mental work. At level of the cortex, precise planning of initiation narrowly looks like to the action executed, is noticed in areas concerned to motor control. This stimulation might oblige as the foundation for the special effects of mental training. A model that is called as, hierarchical model of the organization of action projected the temporary memory storage of a 'copy' of the many demonstrative stages. These retentions vanish when an action associated to the denoted goal takes place. By compare, if the act is partial or not performed, the entire system rest stimulated, and the content of the demonstration is experienced. This mechanism would be the foundation for conscious reach to this content during motor imagery and mental training [5].

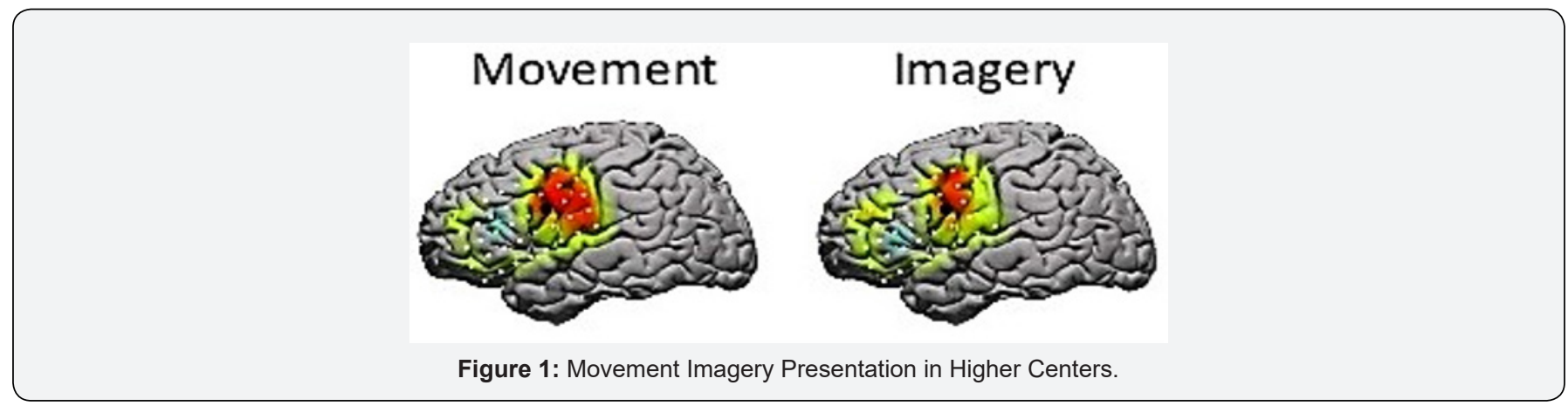


By functional magnetic resonance imaging (FMRI) techniques, the intensity and latitudinal distribution of functional activation in the left pre and post central gyri, during actual motor performance (MP) and mental representation of selfpaced finger-to-thumb opposition movements of the dominant hand were investigated in fourteen right-handed volunteers [6]. For many patients, it is difficult or sometimes impossible to execute motor tasks, who have damaged central nervous system, even after initial involvement in an dynamic rehabilitation platform. It is lately offered by several investigators that these patients can use mental practice as a healing device to increase their motor functions, but very little experiential work reports this problem openly. This article deliberates the reason for inspecting mental practice for encouraging motor recovery in patients who have neurological syndromes. We first showed evidence that supports the presence of a resemblance between performed and imagined activities by using records from psychophysical, neurophysiologic, and brain imaging studies. Then this additionally describes the recurrence of movements through bodily and mental repetition of a mechanical skill. Lastly, a new model is suggested to focus the key role of motor imagery as a crucial method of mental rehearsal, and also to stimulate further research on this type of training in the restoration of patients with motor deficiencies having cerebral origin [7].

There is evidence to suggest that mental practice of movement can produce normal effects recognized as practicing the actual movements. Imagining hand movements could stimulate restoration and redistribution of brain activity, accompanied by recovery of hand function, thus resulting in a reduced motor impairment. Current evidence for mental practice with motor imagery (MI) in stroke is insufficient due to methodological limitations [8] (Figure 1). Mental practice is the intended preparation of mental tasks, whereas motor imagery practice results in motor improvement through motor imagery rehearsal. These both italicized terms are sometimes used interchangeably. So, we will treat them synonymously. MI is cheapest and easily available. It is necessary for physical therapist to be informed about the use of MI, because of increasing number of reports about its benefits of MI in improving motor performance [9]. Mental Rotation Task / Paradigm is a tool to assess the child's ability of mental imagery. It is process of imagining an object rotating in three dimensional spaces. Person is asked to decide if these two figures are the same shape but rotated or they are two different shapes [10] (Figure 2).

(a)

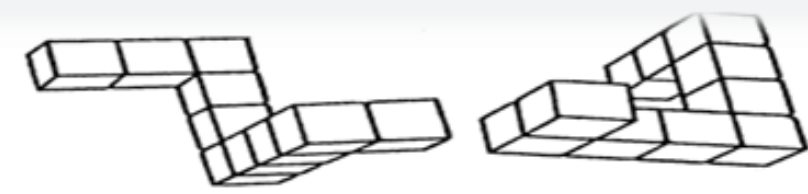

(b)

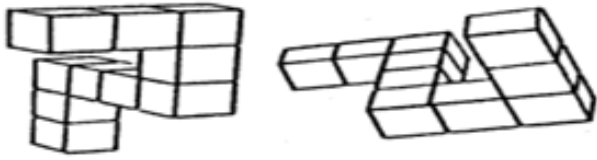

Mental Rotation Test-Are these two figures

Figure 2: Mental Rotation test.

\section{Methodology}

Randomized clinical trial was conducted in department of physical therapy of National Institute of Rehabilitation Medicine, Islamabad, for duration of 6 months. 30 patients with diagnosed with spastic Cerebral Palsy, then 22 participated in this study according to inclusion and exclusion criteria. After selection of patients who fulfilled inclusion criteria, they were arbitrarily divided into two groups by means of simple lottery method. i.e. Control group n=11 and Interventional Group n=11. Baseline assessment was done on 0 week; second Assessment on 4 th week Post-intervention assessment was done after 8 weeks. Total duration of study treatment was 12 weeks. The experimental group performed motor imagery training as specific intervention, that involves motor imagining of gross movements of lower limb $15 \mathrm{~min}$. embedded in conventional therapy for $30 \mathrm{~min}$. (45 min/day, 3 times/week). And, the control group was given conventional therapy only (30 min./day, 3 times/ week).
The interventional group received Motor Imagery training along with conventional therapy. In MI, a small animated Video clips were designed to display and guide the child through imagery process. Motor Imagery Training involves imagining task with external visual stimulus, given via short video clip i.e.

i. Football playing

ii. Running a race

iii. Balancing on one leg

iv. Keeping balance on a wobble board

v. Jumping from block to block

The control group received Conventional Physical Therapy only including,

a) Therapeutic Ball Exercises

b) Balance Board Training 
c) Stretching Exercises

d) Recreational activities.

\section{Results}

The Results of both tools TUF and MFR are significant indicates that Motor Imagery is an effective technique to improve balance and gross motor skills in Spastic Cerebral Palsy whereas tool GMFM results are non-significant indicating that this intervention techniques are not that much effective in case of cerebral palsy.

Overall, the Mean age of all patients was 9.36 \pm 3.170 . and in control group mean and standard deviation values were $9.36 \pm 2.730$ and in experimental group $9.36 \pm 3.695$ were mean and standard deviation values. There were $77.3 \%$ male and $22.7 \%$ female in the study. In experimental group $72.7 \%$ were male and $27.3 \%$ female and in control group $81.8 \%$ were male and $18.2 \%$ female. In general, $31.8 \%$ patients were diagnosed with spastic hemiplegic CP (Rt), $22.7 \%$ patients with spastic hemiplegic CP (Lt), 27.3\% patients with spastic diaplegia while $18.2 \%$ patients with Spastic quadriplegia. In experimental

Table 1: Between Groups Analysis of TUG. group, there were $27.3 \%$ of patients were diagnosed with spastic hemiplegia (Rt), 18.2\% patients with spastic hemiplegic CP (Lt), $36.4 \%$ with spastic diaplegia while $18.2 \%$ were with Spastic quadriplegia. In control group, there were $36.4 \%$ of patients were diagnosed with spastic hemiplegia (Rt), $27.3 \%$ patients with spastic hemiplegic CP (Lt), 18.2\% with spastic diaplegia while $18.2 \%$ were with Spastic quadriplegia.

Then, by relating Q-Q PLOT normality of data was checked, to recognize normal distribution of the data Kolmogorov-Smirnov test indicated that data were not normally distributed for Time up and go test (TUG) test and Modified Functional Reach (MFR) at baseline. Nonparametric test that is Mann Whitney U- Test for between group analysis and wilcoxon test were applied on both TUG and MFR test, but for GMFM-IS66 Independent T test was applied for between group analysis and Repeated measure anova for analysis of time interval within each group was applied for. These tests were used to compare Pre and Post Treatment of between Group A and B. As, the data for TUG and MFR was nonnormally distributed at the baseline so Mann Whitney U Test was applied to compare the all three assessment values between control and experimental group (Tables 1-3).

\begin{tabular}{|c|c|c|c|c|}
\hline \multicolumn{2}{|c|}{ Time up and Go test (TUG) } & Interquartile range (IQ) & Mean rank & P-value \\
\hline \multirow{2}{*}{ Baseline Assessment } & Interventional group & \multirow{2}{*}{$21.90(10)$} & 13.18 & \multirow{2}{*}{0.222} \\
\hline & Control group & & 9.82 & \\
\hline \multirow{2}{*}{$2^{\text {nd }}$ Assessment } & Interventional group & \multirow{2}{*}{$21(9)$} & 12.23 & \multirow{2}{*}{0.598} \\
\hline & Control group & & 10.77 & \\
\hline \multirow{2}{*}{ Final Assessment } & Interventional group & \multirow{2}{*}{$20(9)$} & 11.41 & \multirow{2}{*}{0.947} \\
\hline & Control group & & 11.59 & \\
\hline
\end{tabular}

Table 2: Between Groups Analysis of MFR.

\begin{tabular}{|c|c|c|c|c|}
\hline \multicolumn{2}{|c|}{ Modified Functional Reach Test (MFR) } & Interquartile Range (IQ) & Mean rank & P-value \\
\hline \multirow[t]{2}{*}{ Baseline Assessment } & Interventional group & \multirow[b]{2}{*}{$3.30(1)$} & 10.77 & \multirow[t]{2}{*}{0.596} \\
\hline & Control group & & 12.23 & \\
\hline \multirow{2}{*}{$2^{\text {nd }}$ Assessment } & Interventional group & \multirow[b]{2}{*}{$3.40(0)$} & 13.50 & \multirow[t]{2}{*}{0.075} \\
\hline & Control group & & 8.73 & \\
\hline \multirow{2}{*}{ Final Assessment } & Interventional group & \multirow[b]{2}{*}{$3.60(1)$} & 14.45 & \multirow{2}{*}{0.030} \\
\hline & Control group & & 8.55 & \\
\hline
\end{tabular}

Table 3: Between Groups Analysis of GMFM.

\begin{tabular}{|c|c|c|c|}
\hline \multicolumn{2}{|c|}{ GMFM-IS66 } & Mean \pm SD & P-Value \\
\hline \multirow{3}{*}{ Baseline Assessment } & Interventional group & $67.72 \pm 7.25$ & 0.975 \\
\cline { 2 - 4 } & Control group & $67.80 \pm 4.38$ & 0.975 \\
\hline \multirow{2}{*}{$2^{\text {nd }}$ Assessment } & Interventional group & $66.79 \pm 7.71$ & 0.767 \\
\cline { 2 - 4 } & Control group & $67.59 \pm 4.34$ & 0.768 \\
\hline \multirow{2}{*}{ Final Assessment } & Interventional group & $66.51 \pm 7.90$ & 0.696 \\
\cline { 2 - 4 } & Control group & $67.59 \pm 4.35$ & 0.697 \\
\hline
\end{tabular}

\section{Discussion}

All patients with hemiplegia have difficulty with planning motor movements, Effect of motor imagery on hemiplegic cerebral palsy and on Developmental Congenital Disorder and concluded that anticipatory motor planning was improved by this intervention [11]. Results of this study were supported by 
the study, In 2013 by Bert Steen Bergen et al., on 'Impaired motor planning and motor imagery in children with unilateral spastic cerebral palsy' concluded that Motor imagery training may be a valid and useful tool to complement upper limb rehabilitation in young children with CP, supports researcher's study as Motor imagery techniques were helpful in increasing balance and gross motor function of children with cerebral palsy and showed significant $p$ value $<0.005$ [2]. According to another study in 2012 by Hwai-young Cho et al; on 'Effects of motor imagery training on balance and gait abilities in post-stroke patients: a randomized controlled trial' concluded that if gait training with motor imagery training is used it will recovers the balance and gait of chronic stroke patients more better then gait training alone, that supports researcher's study as Motor imagery techniques were helpful in increasing balance and gross motor function of children with cerebral palsy and showed significant $\mathrm{p}$ value $<0.005[12]$.

According to another study in 2008 by Ayelet et al; 'HomeBased Motor Imagery Training for Gait Rehabilitation of People With Chronic Post stroke Hemiparesis' says that There were noteworthy rises in stride length, cadence, and single-support time of the pretentious lower limb, giving strength to my study as Motor imagery techniques were helpful in increasing balance and gross motor function of children with cerebral palsy and showed significant $\mathrm{p}$ value $<0.005$. According to another study in 2009 by Bert Steenbergen et al; on 'Motor imagery training in hemiplegic cerebral palsy: a potentially useful therapeutic tool for rehabilitation' gives that Motor imagery training may be a valuable additional tool for rehabilitation in children with hemiparetic CP giving strength to my study as Motor imagery techniques were helpful in increasing balance and gross motor function of children with cerebral palsy and showed significant p value $<0.005$ [11]. In 2012, a study by Hwai Young Cho et al., concluded that gait training along with motor imagery training increases the balance and gait capabilities of chronic stroke patients suggestively better than gait training alone thus, giving strength to my study as motor imagery techniques were helpful in growing balance and gross motor function of children up with cerebral palsy and revealed significant $\mathrm{p}$ value $<0.005$.

\section{Conclusion}

The study results concluded that both treatment groups were improved by Physical Therapy. This study suggests that motor imagery techniques combine with conventional combination therapy has significant effects in improvement of gross motor functional skills of children with spastic cerebral palsy, age between 7 and 15 years. There was significant improvement in the balance level whereas, there was little increase in level of gross motor function. So, Motor imagery is an effective technique to improve motor planning and balance among cerebral palsy.

\section{Acknowledgement}

First of all I would like to pay tribute to Almighty ALLAH who helps me in all the even and odd situations during the completion of my research work, without His will I would never be able to do the research project. I would like to express my great appreciation to my research supervisor Dr. Mirza Obaid baig who helped me throughout my research project with his priceless and productive ideas; it was my great pleasure to work with an individual of intellect like him. Then I would like to pay gratitude to my parents without their sincere prayers I would never be able to achieve what I have today. Sincere thanks to all my friends and family members who prayed for me.

\section{References}

1. Kim JY, Kim JM, Ko EY (2014) The effect of the action observation physical training on the upper extremity function in children with cerebral palsy. Journal of exercise rehabilitation 10(3): 176-183.

2. Steenbergen B, Jongbloed-Pereboom M, Spruijt S, Gordon AM (2013) Impaired motor planning and motor imagery in children with unilateral spastic cerebral palsy: challenges for the future of pediatric rehabilitation. Dev Med Child Neurol 55(Suppl 4): 43-46.

3. Coslett HB, Medina J, Kliot D, Burkey AR (2010) Mental motor imagery indexes pain: the hand laterality task. Eur J Pain 14(10): 1007-1013.

4. Schnitzler A, Salenius S, Salmelin R, Jousmäki V, Hari R (1997) Involvement of primary motor cortex in motor imagery: a neuromagnetic study. Neuroimage 6(3): 201-208.

5. Jeannerod M (1995) Mental imagery in the motor context. Neuropsychologia 33(11): 1419-1432.

6. Porro CA, Francescato MP, Cettolo V, Diamond ME, Baraldi P, et al. (1996) Primary motor and sensory cortex activation during motor performance and motor imagery: a functional magnetic resonance imaging study. J Neurosci 16(23): 7688-7698.

7. Jackson PL, Lafleur MF, Malouin F, Richards C, Doyon J (2001) Potential role of mental practice using motor imagery in neurologic rehabilitation. Arch Phys Med Rehabil 82(8): 1133-1141.

8. Ietswaart M, Johnston M, Dijkerman HC, Joice S, Scott CL, et al. (2011) Mental practice with motor imagery in stroke recovery: randomized controlled trial of efficacy. Brain 134(5): 1373-86.

9. Dickstein R, Deutsch JE (2007) Motor imagery in physical therapist practice. Phys Ther 87(7): 942-953.

10. Ionta S, Blanke O (2009) Differential influence of hands posture on mental rotation of hands and feet in left and right handers. Exp Brain Res 195(2): 207-217.

11. Steenbergen B, Crajé C, Nilsen DM, Gordon AM (2009) Motor imagery training in hemiplegic cerebral palsy: a potentially useful therapeutic tool for rehabilitation. Developmental Medicine \& Child Neurology 51(9): 690-696.

12. Dunsky A, Dickstein R, Marcovitz E, Levy S, Deutsch J (2008) Homebased motor imagery training for gait rehabilitation of people with chronic poststroke hemiparesis. Archives of physical medicine and rehabilitation 89(8): 1580-1588. 
CC (i) This work is licensed under Creative Commons Attribution 4.0 Licens

BY DOI: $10.19080 / J P F M T S .2019 .06 .555696$
Your next submission with Juniper Publishers will reach you the below assets

- Quality Editorial service

- Swift Peer Review

- Reprints availability

- E-prints Service

- Manuscript Podcast for convenient understanding

- Global attainment for your research

- Manuscript accessibility in different formats

( Pdf, E-pub, Full Text, Audio)

- Unceasing customer service

Track the below URL for one-step submission

https://juniperpublishers.com/online-submission.php 